\title{
Variability of pharmacogenomic information in drug labels approved by different agencies and its ethical implications
}

\author{
Müberra Güner ${ }^{1}$, Perihan Elif Ekmekci ${ }^{1}$, and Berra Kurtoglu ${ }^{1}$ \\ ${ }^{1}$ TOBB Economics and Technology University
}

September 10, 2020

\begin{abstract}
Rationale, aims and objectives: The efficacy and safety of drugs can be improved by rational prescription and personalization of medicine for each patient. Pharmacogenomics information (PGx) in drug labels (DL) is important for the personalization of medications because genetic differences may affect both drug efficacy and safety. Providing adequate PGx to patients has ethical implications. The aim of this study was to determine if there are discrepancies among various agency-approved labels for the same active ingredient and where the labels approved by the Turkish Medicines and Medical Devices Agency (TMMDA) stand regarding the inclusion of PGx and discuss these ethical implications. Methods: DL annotations from the Pharmacogenomics Knowledgebase and DLs approved by the TMMDA were analyzed according to information and action levels, which are "testing required", "testing recommended", "actionable", and "informative". Results: There are 381 drugs listed in PharmGKB drug label annotations and 278 of these have biomarkers. A total of $242(63.52 \%)$ drugs are approved and available in Turkey. Of these, $207(54.33 \%)$ contain the same information as in or similar to that in the labels approved by the other agencies. The presence and level of information varied among the DLs approved by different agencies. The inconsistencies may have a significant effect on the efficacy and the safety of drugs. Conclusion: These findings suggest an urgent need for the standardization of pharmacogenomics information globally because it may not only affect the efficacy and safety of medications but also essential ethical rules regarding patient rights.
\end{abstract}

\section{Introduction}

Pharmacogenomics has been described as the study of differential responses to pharmacological treatment due to heredity, which has drawn attention to personalized medicine [1-5]. The genetically determined interindividual variability in enzyme activity, receptors, transporters, and various other drug targets has been associated with the efficacy and toxicity of pharmaceutical treatment [6-9]. Polymorphisms, mutations, gene expression differences, and chromosomal abnormalities that cause changes in these targets may be the main reason for variations in the body's reaction to drug exposure. Variability in drug efficacy and safety is explained by pharmacogenomics besides factors such as age, sex, body mass index, renal and liver functions, other diseases, and lifestyle differences [10-14].

Inter-individual variations in drug responses not only affect drug efficacy but also can lead to adverse drug reactions (ADRs) in patients. ADRs are associated with morbidity and mortality and are a burden to the healthcare system, accounting for a cost of almost 100 billion USD [7]. According to a prospective analysis, up to $6.5 \%$ of acute hospital admissions in the United Kingdom are still related to ADRs [16].

ADRs can be minimized by rational prescription and personalization of medicine for each patient, the precision of which can be increased by considering the pharmacogenomic information (PGx) during these processes. The European Commission published a guideline on the summary of product characteristics (SmPCs) in 2009, which emphasized that all available relevant PGx should be included in the SmPCs [17]. In 2015, a review of 517 pharmaceutical products approved for use by the European Medicines Agency 
(EMA) from 1995 to 2014 showed that the SmPCs of approximately 15\% of medications consisted of PGx that directly impacted treatment [18]. Similarly, Frueh et al. performed a review of the labels of the drugs approved by the United States Food and Drug Administration (FDA) and estimated that almost one-fourth of patients in 2006 (approximately 8.8 million) were prescribed one or more drugs for which the label included PGx in the US [19]. We estimate that this number has increased considerably over time.

Providing adequate PGx of the drugs to patients has some ethical implications in terms of providing benefits, avoiding harm, mitigating risks, strengthening the trust in healthcare providers, and empowering patients, as the new-found scientific information in pharmacogenomics increases the moral responsibility to effectively transfer this information to patients. Therefore, the aim of this study was to determine if there are discrepancies among various agency-approved labels for the same active ingredient and where the labels approved by the Turkish Medicines and Medical Devices Agency (TMMDA) stand regarding the inclusion of PGx and discuss the ethical implications of SmPCs with inadequate and outdated information in the context of the principles of medical ethics.

\section{Methods}

We downloaded all data on 14 April 2020, from which we extracted the level tag and PGx. The SmPCs TMMDA-approved drugs retrieved from the agency's website and checked and updated according to the 2020 version of RX Media Pharma Interactive Pharmaceuticals Information Source were screened and analyzed for PGx content [20,21]. Then, we compared the information provided in TMMDA-approved labels with that in the Pharmacogenomics Knowledgebase (PharmGKB) database [22]. PharmGKB annotates drug labels containing PGx approved by the FDA, EMA, Swiss Agency of Therapeutic Product (Swissmedic), Pharmaceuticals and Medical Devices Agency, Japan (PMDA), and Health Canada (Santé Canada, HCSC). PharmGKB annotations provide a brief summary of the PGx in the label, an excerpt from the label, and a downloadable highlighted label PDF file. A list of genes and phenotypes found within the label is mapped to label section headers and listed at the end of each annotation. PharmGKB also attempts to interpret the level of action implied in each label with the "PGx Level" tag [22]. There are four levels of actions:

1. Testing required, states or implies that some sort of gene, protein, or chromosomal testing, including genetic testing, functional protein assays, and cytogenetic studies, should be conducted before using this drug. 2. Testing recommended, states or implies that some sort of gene, protein or chromosomal testing, including genetic testing, functional protein assays, and cytogenetic studies, is recommended before using this drug. 3 . Actionable PGx, may contain information about changes in efficacy, dosage, metabolism, or toxicity due to gene/protein/chromosomal variants or phenotypes (e.g., "poor metabolizers"), or the label may mention contraindications of the drug in a particular subset of patients with particular variants/genotypes/phenotypes. 4. Informative PGx, contains information stating that particular gene/protein/chromosomal variants or metabolizer phenotypes do not affect a drug's efficacy, dosage, metabolism, or toxicity, or states that particular variants or phenotypes affect a drug's efficacy, dosage, metabolism, or toxicity, but this effect is not "clinically" significant. Or, the label appears or appeared on the FDA Biomarker List but does not currently meet the requirements to be assigned as "Testing required," "Testing recommended," or "Actionable PGx."

First being the highest level, we analyzed the consistency of recommendations of labels approved by different agencies as well.

Statistical analysis: The data were presented as the percentage of the drug labels approved by each agency and presented as numbers and percentages. No statistical comparison was performed as the aim of this study was to determine the discrepancies among agencies and the possible effects of these discrepancies on ethical issues.

\section{Results}

There are 381 drugs listed in PharmGKB drug label annotations and 278 of these have biomarkers. The numbers of drug labels containing PGx approved by the FDA, EMA, Swissmedic, HCSC, and PMDA are 306 (80.32\%), $127(33.33 \%), 125(32.81 \%), 104(27.30 \%)$, and $52(13.65 \%)$, respectively. A total of $242(63.52 \%)$ 
drugs are approved and available in Turkey. Of these, 207 (54.33\%) contain the same information as in or similar to that in the labels approved by the other agencies. Some labels contain two levels of information if different genes are involved and/or if the drug is a combination of two or more active ingredients. The details of the information levels are shown in Table 1.

One hundred ninety-one (50.13\%) of the drugs had labels approved by more than one agency, and the levels of PGx were inconsistent for $63(32.98 \%)$ of these drugs. For example, in one country label, the information level is testing required while that in the others is testing recommended or actionable or informative information. Among the 132 drugs that are not currently on the Turkish market, the labels of 55 (41.67\%) have no PGx at all. Twenty-three (17.42\%) of these drugs are on the import drug list. This list contains the drugs that are not currently licensed and/or not marketed in Turkey but their import in line with a set of regulations is permitted by the Turkish Ministry of Health. Of the 23 drugs that are on the import list, three have SmPCs in Turkish and two in English. Eighteen (13.63\%) are licensed but are currently unavailable due to not being produced, not being imported, or not being marketed. Of these, $11(61.11 \%)$ have PGx in their approved SmPCs. RX Media Pharma database monographs for active ingredients contain the PGx for $46(34.85 \%)$ of the drugs.

\section{Examples of inconsistencies}

\section{Types of human leukocyte antigen}

Thirteen drug label annotations contain information about various human leukocyte antigen (HLA) types. Some of the inconsistencies are reasonable because the frequencies of HLA types vary among different populations. For example, all agencies but the PMDA requires testing for HLA-B*5701 before therapy with abacavir. The information in the PMDA-approved label is informative because the prevalence of this allele is only $0.1 \%$ in the Japanese population.

For carbamazepine, while the FDA- and Swissmedic-approved labels require screening of patients with ancestry in line with genetically at-risk populations (individuals of Asian descent) for the presence of the HLA-B*1502 allele prior to treatment due to a high risk of serious, and sometimes fatal, dermatologic reactions, HCSC and TMMDA recommend testing. PMDA's level of information is only actionable and states that the HLA-B*1502 allele was prevalent in nearly all of a group of Han Chinese patients who developed StevenJohnsons Syndrome or toxic epidermal necrolysis (SJS/TEN). Swissmedic and HCSC also recommend testing for HLA-A*3101, which increases the risk of developing hypersensitivity reactions to carbamazepine. On the other hand, the FDA and PMDA levels of information are actionable for this allele, despite the fact that it appeared at a high frequency in Japanese patients. Similarly, for oxcarbamazepine, the Swissmedic-approved label requires testing for the HLA-B*1502 allele as due to the increased risk of SJS/TEN, the FDA and TMMDA recommend testing. Information is more variable in the case of phenytoin and HLA-B*15:02. The FDA and Swissmedic level of information is actionable and states the high risk of SJS/TEN in patients of Asian ancestry. The HCSC recommends testing and the TMMDA has an informative level of information for three of the five generic formulations. Although Han/Chinese descendants are rare in Turkey's population, the inconsistency in different pharmaceutical/commercial forms is notable.

\section{Glucose 6 phosphate dehydrogenase deficiency}

Sixty drug labels that have PGx on risks and complications when the drugs are used in glucose 6 phosphate dehydrogenase (G6PD)-deficient patients. Fifty-three of these drugs have an actionable level of information (such as contraindicated in patients with G6PD deficiency), three have an informative level of information (e.g., succimer has been used for the treatment of lead poisoning in five patients with G6PD deficiency without adverse reactions; patients with a known history of G6PD deficiency was excluded from some clinical trials; or patients with a known history of G6PD deficiency was excluded from some clinical trials), and two have testing required level of information (tafenoquine and pegloticase) for all agencies. The labels for primaquine labels were inconsistent: the FDA-approved drug label for primaquine states that G6PD testing should be performed before using the drug and that the drug should not be prescribed for patients with severe G6PD deficiency. However, the HCSC-approved product monograph information level was actionable for 
primaquine and states that caution should be exercised while using the drugs in individuals with G6PD deficiency due to the risk of hemolytic anemia, which we assume is based on the previous testing. Similarly the information levels for rasburicase are also variable: the FDA-approved label has and testing required and states that patients at higher risk for G6PD deficiency, such as those of African or Mediterranean ancestry, should be screened prior to starting treatment, because rasburicase is contraindicated in patients with G6PD deficiency. The EMA-, Swissmedic-, and PMDA-approved labels were actionable and stated that rasburicase is contraindicated in patients with G6PD deficiency due to risk of hemolytic anemia, which we again believe is based on the assumption that the patient would have the information on G6PD status prior to drug use. The HCSC-approved label level was testing recommended and states that patients at higher risk for G6PD deficiency (e.g., those of African or Mediterranean ancestry) be screened prior to starting treatment, due to the risk of severe hemolytic anemia.

TMMDA had actionable information in 17 and informative information in three of the drug labels. For seven of the drugs (ceftriaxone, ciprofloxacin, glibenclamide, gliclazide, nitroglycerin, sulfamethoxazole/trimethoprim, and vitamin C), the information in the labels differs between the original and/or generic forms.

\section{Cytochrome P 450 enzymes}

Of the 381 drugs, the labels of 143 have information on various cytochrome P 450 enzymes of varying levels: actionable, 68 (47.55\%); informative, 47 (32.87\%); testing required, 6 (4.20); and testing recommended, 1 $(0.70 \%)$. The labels of the remaining 21 drugs $(14.69 \%)$ have varying levels of information. The information was about the enzymes CYP2D6 $(\mathrm{n}=90,54.55 \%)$, CYP2C19 $(\mathrm{n}=26,15.76 \%)$; CYP3A4 $(\mathrm{n}=23,13.94 \%)$, CYP2C9 $(\mathrm{n}=17 ; 10.30 \%)$, and others $(\mathrm{n}=7,4.24 \%)$.

Fifteen drugs with the information level informative have been approved by the EMA. However, the information states that "no pharmacogenomics information is available". Information on the role of enzymes or proteins that are involved in the pharmacokinetics of the drug and that may lead to drug-drug interactions if concomitantly used with other drugs using the same enzymes or proteins during pharmacokinetic activities are included in these labels. There are two examples of different levels of information provided by other agencies (if any), indacaterol and voriconazole.

For indacaterol, the information in the FDA- and Swiss-approved labels is also informative but more detailed and states the genotypes that may lead to pharmacokinetic changes. On the other hand, the information in the PMDA-approved label is classified as actionable although it is similar to that provided by other agencies. The labels actually contain the same information but in worded differently.

The EMA European Public Assessment Report (EPAR) for indacaterol (Hirobriz Breezhaler) does not contain PGx but does contain information regarding the drug's pharmacodynamics as a beta2-adrenoceptor (ADRB2) agonist and pharmacokinetics involving UGT1A1, CYP3A4, and ABCB1.

The PMDA package insert for indacaterol (Onbrez) states that individuals with low UGT1A1 expression have increased steady-state area under the concentration-time curve (AUC) and maximum plasma concentration (Cmax) compared to those among individuals with wild-type UGT1A1 expression. Following repeated inhalation of indacaterol in subjects with a variant, low-activity UGT1A1 genotypes, the steady-state Cmax and AUC were both 1.2-fold higher than those related to the wild-type genotype (non-Japanese data).

The FDA-approved drug label for indacaterol (ARCAPTA NEOHALER) states that the steady-state AUC and Cmax of indacaterol were 1.2 -fold higher in patients with the (TA) $7 /(\mathrm{TA}) 7\left({ }^{*} 28 /{ }^{*} 28\right)$ genotype than in the patients with the $(\mathrm{TA}) 6 /(\mathrm{TA}) 6\left({ }^{*} 1 /{ }^{*} 1\right)$ genotype, suggesting no relevant effect of the UGT1A1 genotype on indacaterol exposure.

The information is actionable for voriconazole labels approved by other agencies. The EMA-approved label states that it does not contain PGx; it contains warning information regarding the co-administration of drugs that are substrates, inhibitors, or activators of CYP3A4, CYP2C9, or CYP2C19 due to drug-drug interactions. The actionable information for the same drug states that CYP2C19 poor metabolizers have, 
on average, four-fold higher voriconazole exposure than do normal metabolizers. In contrast, intermediate metabolizers have two-fold higher exposure than do normal metabolizers. The PMDA-approved label notes that voriconazole is metabolized by CYP2C19 (as well as CYP2C9 and CYP3A4) and provides tables showing the difference in pharmacokinetic metrics between extensive, intermediate, and poor metabolizers in adults and children.

Ninety-three TMMDA-approved drug labels have information about cytochrome P 450 enzymes. Of these, the labels of $41(44.09 \%), 31(33.33 \%)$, and $1(1.08 \%)$ drug contain informative information, actionable information, and state testing recommended, respectively. Twenty (21.51\%) labels contained information on drug-drug interactions and concomitant use with enzyme inducers or inhibitors. However, we did not classify these as informative labels, although some of the EMA-approved labels were similar to informative labels. For five drugs, the original drug labels had information similar to that in the corresponding labels approved by other agencies; however, this similarity was lacking among the labels for the generic versions of these drugs.

\section{Discussion}

According to licensing regulations, approved licenses are valid for 5 years. There are no specific regulations about SmPCs, which are included in the license application files and updated accordingly. Global pharmaceutical companies update their licenses and SmPCs in a timely manner when they have new information. However local and/or generic companies do not update their licenses and SmPCs strictly according to these rules. Furthermore, most discrepancies are observed in the case of generic or older products.

When we evaluated the TMMDA-approved labels, we found that the labels available were in accordance with those approved by other agencies, mainly the EMA and FDA. The two major problems concerning the pharmacovigilance information pertaining to the Turkish pharmaceutical market are that generics may not have the same/similar information as the original drugs and imported drugs lack information in Turkish for the patient and/or for the prescriber.

Improvements in pharmacogenomics have enhanced its applications in medical interventions. Therefore, it is necessary to disclose PGx; however, the content communicated and the process of informing patients about pharmacogenomics remains tentative [23]. Nevertheless, our study reveals the fact that SmPCs are not standardized and do not necessarily contain up to date information. A review of the literature shows that this is not a local problem peculiar to some countries such as Turkey, and is, in fact, a global problem [24-27]. SmPCs are suggested as useful and effective tools for disclosing PGx [28]. However, because of inadequate, inconsistent, and outdated information, SmPCs are not appropriate for providing the suggested information to patients [29].

The scarcity of regulation pertaining to the PGx to be included in SmPCs and standardized processes and the reluctance of pharmaceutical companies in updating SmPCs have some widespread ethical implications $[27,30,31]$.

1. The first ethical implication is about the right of the patient to be informed about the medication that is being given to the patient. The right to have access to accurate and relevant information emerges from the basic human right of autonomy [32]. Respect for autonomy is one of the main principles of medical ethics and it is realized by disclosing the necessary information to the patient and providing space for patients' decision-making without the undue influence of others. Disclosure of information is a complex procedure that does not start and end in one instance, but rather is an ongoing process that begins with the interaction between the physician and the patient and continues throughout the treatment. Patients may not be able to understand the disclosed information in clinical settings, because several factors might impede their cognitive capacity in that clinical setting or the patients may have trouble recalling the disclosed information later when they leave the hospital or need to refresh their memories upon achieving an unexpected result with the medication [33,34]. Compared to other medical information, PGx may be more confusing for patients. Thus, we can assume it would be harder to understand or recall what has been disclosed to patients [35]. SmPCs are useful references for patients in this respect and should be regarded as a part of the process of 
information disclosure to patients. Neglecting to update SmPCs hampers the right of patients to have access to accurate information and prevents them from exercising their autonomy.

2. Another ethical issue emerges from the principle of doing no harm to the patient. SmPCs with outdated information may cause harm by misleading patients about their medications. The principle of doing no harm encumbers responsibility to all parties involved in health care provision [32]. Pharmaceutical companies have a significant role in health care services; hence, they are obliged to comply with the principle of "do no harm" of medical ethics. The context of this principle is not limited to not doing any intentional harm but has a broader context to cover risk mitigation for the patient. Pharmaceutical companies that provide SmPCs with poor content fail to acknowledge this principle.

3. Not having adequate and consistent PGx in SmPCs poses a high risk for harm among vulnerable groups such as children, the elderly, or pregnant women. Since these population groups are considerably less subject to clinical trials in pharmacogenomics, the accumulated scientific data are limited. Moreover, a lack of clarity about the differences in pharmacogenomic research for discovering medical interventions and optimizing clinical care makes the provision of information to these patient groups particularly important [36]. The pharmacogenomics counseling skills for communicating with vulnerable populations and the knowledge and interest of healthcare providers in new-found PGx are variable and limited depending on their areas of expertise [37-39]. Hence well-written SmPCs may provide significant benefits and avoid harm among these vulnerable groups.

4. Veracity and reliability are two important ethical values in clinical ethics. Patients should trust that their healthcare providers will do their best to benefit patients' health. Trust in the physician is the first ring in the chain and should be backed by trust in the health care system and pharmaceutical companies. Trust is gained by veracity and reliability between the parties. A lack of SmPCs or of SmPCs with different content may harm the trust in health care agencies and pharmaceutical companies by raising concerns about sloppy or inattentive process management. It is plausible to argue that ruining patients' trust in the healthcare system is another way of doing harm to patients, which would impair the success of their treatment in the long term.

5. Patient empowerment is an essential component of personalized medicine. Empowered patients play a new, more proactive, and leading role in the management of their health status. Recently direct-to-consumer (DTC) DNA testing companies have been offering PGx to patients, and they will potentially be more at the forefront of delivering personalized medicine in the near future [40]. Providing PGx of drugs by means of SmPCs to patients presumably contributes to patient empowerment by raising patients' awareness about their medical conditions and their proactivity during the course of treatments.

Based on the findings of our study, the standard of PGx in SmPCs is far from that required for meeting these essential ethical requirements. It is clear that an international organization such as the International Council for Harmonization should act as a guide to make essential PGx available and standardized globally. National agencies need to agree on the definition of PGx and its levels. PharmGKB sets a good example in this regard. After an agreement has been reached on the standardization of PGx for inclusion in SmPCs, marketing license owners need to check the approved labels, find discrepancies, and change labels accordingly. Similarly, generic companies must be subject to the same requirements: they should be required to update their SmPCs and other package inserts. Following these simple recommendations will not only prevent the ethical breach of patients' rights but also will equip physicians to precisely personalize the medicine being prescribed.

\section{References:}

1. Bishop JR. Pharmacogenetics.Handb Clin Neurol. 147:59-73 (2018).

2. Nebert DW. Pharmacogenetics and pharmacogenomics: why is this relevant to the clinical geneticist? Clinical Genetics. 56: 247-258 (1999).

3. European Medicine Agency. Position paper on terminology in pharmacogenetics discussion at the pharmacogenetics expert group. Eur Med Agency - Comm Propr Med Prod [Internet]. (November):0- 
6. (2001) Available from: http://www.emea.eu.int. Accessed on 28 May 2020.

4. Jain KK. Personalized medicine. Curr Opin Mol Ther. 4(6):548-58 (2002).

5. Mirsadeghi S, Larijani B. Personalized Medicine: Pharmacogenomics and Drug Development.Acta Med Iran . 55(3):150-165 (2017).

6. Meyer UA. Drugs in special patient groups: clinical importance of genomics in drug effects. In: Carruthers GS, Hoffmann BB, Melmon KL, Nierenberg DW, eds. New York: McGrawHill, 1179-205 (2000).

7. Ingelman-Sundberg M. Pharmacogenetics: an opportunity for a safer and more efficient pharmacotherapy. J Intern Med 250(3):186-200 (2001).

8. Evans WE, Relling MV. Pharmacogenomics: translating functional genomics into rational therapeutics. Science 286: 487-91 (1999).

9. Roses AD. Pharmacogenetics and future drug development and delivery. Lancet 355: 1358-61 (2000).

10. Ingelman-Sundberg M, Sim SC, Gomez A, Rodriguez-Antona C. Influence of cytochrome P450 polymorphisms on drug therapies: pharmacogenetic, pharmacoepigenetic and clinical aspects. Pharmacol Ther 116(3):496-526 (2007).

11. Food and Drug Administration. Table of pharmacogenomic biomarkers in drug labels [Internet] Springfield, MD: The Administration; Jun 62013 [cited 5 May 2020]. https://www.fda.gov/drugs/scienceand-research-drugs/table-pharmacogenomic-biomarkers-drug-labeling Accessed on 28 May 2020.

12. Shoshi A, Muller U, Shoshi A, Ogultarhan V, Hofestadt R. KALIS - An eHealth System for Biomedical Risk Analysis of Drugs. Stud Health Technol Inform. 236:128-135 (2017).

13. Weinshilboum RM, Wang L. Pharmacogenomics: Precision Medicine and Drug Response. Mayo Clin Proc. 92(11):1711-1722 (2017).

14. Eichelbaum M, Ingelman-Sundberg M, Evans WE. Pharmacogenomics and individualized drug therapy.Annu Rev Med . 57:119-137 (2006).

15. Shepherd G, Mohorn P, Yacoub K, May DW. Adverse drug reaction deaths reported in United States vital statistics, 1999-2006. Ann Pharmacother . 46(2):169-175 (2012).

16. Pirmohamed M, James S, Meakin S, et al. Adverse drug reactions as cause of admission to hospital: prospective analysis of 18820 patients. BMJ (Clinical research ed.) 329(7456), 15-19 (2004).

17. Guideline on summary of product characteristics (SmPC). European commission enterprise and industry directorate-general. European Commission. https://ec.europa.eu/health/ sites/health/files/files/eudralex/vol-2/c/smpc_guideline_rev2_en.pdf. (2009). Accessed 5 May 2020.

18. Ehmann F, Caneva L, Prasad K, et al. Pharmacogenomic information in drug labels: European Medicines Agency perspective. Pharmacogenomics J. 15(3):201-10. doi: 10.1038/tpj.2014.86 (2015).

19. Frueh FW, Amur S, Mummaneni P, et al. Pharmacogenomic biomarker information in drug labels approved by the United States Food and Drug Administration: prevalence of related drug use. Pharmacotherapy. 28(8):992-998 (2008).

20. The SmPC of the drugs approved by TDMDA, https://www.titck.gov.tr/kubkt. Accessed on 14 April 2020 .

21. RX Media Pharma Interactive Pharmaceuticals Information Source https://www.eczanet.com/. Accessed on 14 April 2020.

22. PharmGKB Drug label annotations. https://www.pharmgkb.org/labelAnnotations. Accessed on 14 April 2020.

23. Haga SB, Mills R, Moaddeb J. Pharmacogenetic information for patients on drug labels.Pharmgenomics Pers Med. 7:297-305 (2014).

24. Pai SA, Kshirsagar N. A Critical Evaluation of Pharmacogenetic Information in Package Inserts for Selected Drugs Marketed in India and Its Comparison With US FDA-Approved Package Inserts. $J$ Clin Pharmacol. 56(10):1232-42 (2016).

25. Reis-Pardal J, Rodrigues A, Rodrigues E, Fernandez-Llimos F. Comparing cytochrome P450 pharmacogenetic information available on United States drug labels and European Union Summaries of Product Characteristics.Pharmacogenomics J. 17(6):488-493 (2017).

26. Schuck RN, Grillo JA. Pharmacogenomic Biomarkers: an FDA Perspective on Utilization in Biological 
Product Labeling. AAPS J.18(3):573-7 (2016).

27. Zhu Q, Freimuth RR, Pathak J, Durski MJ, Chute CG. Disambiguation of PharmGKB drug-disease relations with NDF-RT and SPL. J Biomed Inform. 46(4):690-6 (2013).

28. Preskorn SH, Hatt CR. How pharmacogenomics (PG) are changing practice: implications for prescribers, their patients, and the healthcare system (PG series part I). J Psychiatr Pract. 19(2):142-9 (2013).

29. Haga SB, Thummel KE, Burke W. Adding pharmacogenetics information to drug labels: lessons learned.Pharmacogenet Genomics. 16(12):847-54 (2006).

30. Shekhani R, Steinacher L, Swen JJ, Ingelman-Sundberg M. Evaluation of Current Regulation and Guidelines of Pharmacogenomic Drug Labels: Opportunities for Improvements. Clin Pharmacol Ther. 107(5):1240-1255 (2019).

31. Tan-Koi WC, Leow PC, Teo YY. Applications of pharmacogenomics in regulatory science: a product life cycle review. Pharmacogenomics J. 18(3):359-366 (2018).

32. Beauchamp TL, Childress JF. Principles of biomedical ethics. 5th ed. New York Oxford University Press; 57-103 (2001).

33. Brezis M, Israel S, Weinstein-Birenshtock A et al. Quality of informed consent for invasive procedures. Int J Qual Health Care . 20(5):352-357 (2008).

34. Kureshi F, Jones PG, Buchanan DM, Abdallah MS, Spertus JA. Variation in patients' perceptions of elective percutaneous coronary intervention in stable coronary artery disease: cross sectional study. BMJ 349: g5309 (2014).

35. Rose D, Russo J, Wykes T. Taking part in a pharmacogenomic clinical trial: assessment of trial participants understanding of information disclosed during the informed consent process. BMC Med Ethics. 14:34 (2013).

36. Madadi P. Ethical Perspectives on Translational Pharmacogenetic Research Involving Children.Pediatr Drugs 17:91-95 (2015).

37. Zakinova A, Long-Boyle JR, French D, et al. A Practical First Step Using Needs Assessment and a Survey Approach to Implementing a Clinical Pharmacogenomics Consult Service. J Am Coll Clin Pharm. 2(3):214-221 (2019).

38. Elewa H, Alkhiyami D, Alsahan D, Abdel-Aziz A. A survey on the awareness and attitude of pharmacists and doctors towards the application of pharmacogenomics and its challenges in Qatar. $J$ Eval Clin Pract. 21(4):703-709 (2015).

39. Amara N, Blouin-Bougie J, Bouthillier D, Simard J. On the readiness of physicians for pharmacogenomics testing: an empirical assessment.Pharmacogenomics J. 18(2):308-318 (2018).

40. Chua EW, Kennedy MA. Current state and future prospects of direct-to-consumer pharmacogenetics.Front. Pharmacol. 3:152 (2012).

\section{Hosted file}

Table_1.docx available at https://authorea.com/users/355479/articles/478685-variabilityof-pharmacogenomic-information-in-drug-labels-approved-by-different-agencies-and-itsethical-implications 